\title{
Immunogenicity after CoronaVac vaccination
}

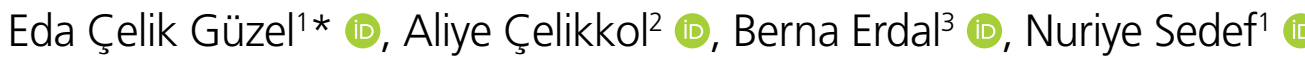

\section{SUMMARY}

OBJECTIVE: This study aimed to investigate the seropositivity of CoronaVac-SinoVac vaccination in severe acute respiratory syndrome coronavirus 2 (SARS-CoV-2) risk factors and comorbidities.

METHODS: Immunoglobulin (IgG) antibody responses were examined on the 21 st day after the second dose of CoronaVac-SinoVac $6 \mu \mathrm{g}$ vaccine on the 28th day. SARS-COV-2 IgG antibody levels were measured by using the enzyme-linked immunosorbent assay method in vaccinated health care workers $(n=134)$ (Group I), vaccinated polymerase chain reaction (PCR) (+) who had coronavirus-19 (COVID-19) disease ( $n=21)$ (Group II), and unvaccinated PCR (+) ( $n=28)$ (Group III) participants. Subgroups were formed in Group I according to the presence of COVID-19 risk factors and comorbidities (diabetes mellitus, cardiovascular disease, and asthma/allergy) and demographic data. RESULTS: Seropositivity rates were 95.5, 100, and $89.3 \%$ for Groups I, II, and III, respectively. IgG antibody levels were found significantly higher in the group between the ages of 20-30 in group I compared to those aged 31-50 and over 50 (both $p<0.01$ ). It was found significantly higher in normal-weight individuals than in the overweight and obese group (both $p<0.01$ ). IgG antibody levels were found significantly lower in people with cardiovascular disease and diabetes mellitus compared with those who did not ( $p<0.05$ and $p<0.001$, respectively). There was a negative correlation between IgG antibody response values and body mass index and age in Group I $(r=-0.336, p<0.001$ and $r=-0.307, p<0.001$, respectively).

CONCLUSION: IgG antibody values decrease with age and with increasing body mass index. The presence of comorbidities (i.e., diabetes mellitus and cardiovascular disease) decreased COVID-19 IgG antibody values.

KEYWORDS: COVID-19 virus disease. Risk factors. Comorbidity. Immunogenicity, vaccine.

\section{INTRODUCTION}

Coronavirus-19 disease (COVID-19) pandemic caused by severe acute respiratory syndrome coronavirus-2 (SARS-CoV-2) has infected more than 110 million people and killed approximately 2.5 million people as of March $2021^{1}$.

Post-vaccine antibody tests against SARS-CoV-2 showed that adequate levels of neutralizing anti-SARS-CoV-2 S1-specific immunoglobulin (anti-S-IgG) antibodies were formed ${ }^{2-5}$. One of these vaccines is the CoronaVac-SinoVac (inactivated
SARS-CoV-2) (Sinovac Life Sciences, Beijing, China) vaccine, whose phase 3 trials have been completed. Phase 1 clinical trials were conducted as dose escalation, and the highest antibody response occurred after two doses of $6 \mu \mathrm{g}$ vaccine $(0$ and 28 days). In the same study, in which phase 2 clinical trials were conducted to increase vaccine production capacity, the seroconversion of neutralizing antibodies was $97 \%$ in the $3 \mu \mathrm{g}$ group and $100 \%$ in the $6 \mu \mathrm{g}$ group. However, the titers of neutralizing S IgG produced after vaccination were lower than the

\footnotetext{
${ }^{1}$ Tekirdağ Namik Kemal University, Faculty of Medicine, Department of Family Physician - Tekirdag, Turkey.

${ }^{2}$ Tekirdağ Namik Kemal University, Faculty of Medicine, Department of Medical Biochemistry - Tekirdag, Turkey.

${ }^{3}$ Tekirdağ Namik Kemal University, Faculty of Medicine, Department of Medical Microbiology - Tekirdag, Turkey.

*Corresponding author: celikguzel@gmail.com

Conflicts of interest: the authors declare there are no conflicts of interest. Funding: none.

Received on May 29, 2021. Accepted on August 16, 2021.
} 
serum S IgG levels of those who had the disease ${ }^{4}$. This is a disadvantage of inactivated vaccines and may affect the response to vaccination of patients in the risk groups for COVID-19.

The aim of this study was to monitor the anti-spike IgG antibody response after CoronaVac vaccination against COVID-19 disease and to investigate the antibody response based on risk factors associated with COVID-19 disease and comorbid conditions.

\section{METHODS}

\section{Study design and participants}

This prospective study was conducted on approval of the Ethics Committee (Protocol number; 2021.55.02.18). In this study, we included 183 volunteers who applied for the vaccination in University Hospital. CoronaVac-SinoVac vaccine was administered intramuscularly in two doses of $6 \mu \mathrm{g}(0$ and 28 days). People who applied for the vaccination in the outpatient clinic for the second dose 28 days following the first dose and whose blood sample was obtained 21 days after the second dose ( $\mathrm{n}=134)$ constituted Group I. PCR (+) patients who have had the disease and also had the first and second dose of the vaccine constituted Group II $(\mathrm{n}=21)$. PCR $(+)$ patients who have had COVID-19 disease but are unvaccinated $(n=28)$ constituted Group III. To examine the IgG antibody response, $2 \mathrm{~mL}$ of whole blood samples were taken 21 days after the second dose of the vaccine from Groups I and II, and immediately from Group III. After centrifugation at 3,000 rpm for $10 \mathrm{~min}$ without delay, the samples were stored at $-80^{\circ} \mathrm{C}$ until further analysis. A COVID-19 positive group (Groups II and IIII) was formed of patients whose quantitative reverse transcription PCR (RT-qPCR) results were positive. Sample collection time was within two months on average from a positive PCR result. Group I was divided into three subgroups with age as 20-30 years, 31-50 years, and above 50 years old, and with body mass index (BMI) as normal (18.5-24.9), overweight (25-29.9), and obese (>30). Smoking, vitamin supplement, regular exercise, and regular sleep characteristics of group I were obtained through a questionnaire. As shown in Table 1, participants were grouped with chronic disease, asthma, diabetes mellitus (DM), cardiovascular disease (CVD), diagnosis, or/and treatment in the last year obtained from the hospital electronic archive and viral load according to working place in the hospital.

Anti-SARS-CoV-2 human IgG measurement was performed using the enzyme-linked immunosorbent assay (ELISA) kit (DIA. PRO, Sesto San Giovanni, Milan, Italy). Serum samples were incubated in wells pre-coated with recombinant nucleocapsid

Table 1. Demographic characteristics of vaccinated participants, Eda Celik Guzel.

\begin{tabular}{|c|c|c|c|}
\hline & $\begin{array}{l}\text { Group I } \\
(n=134)\end{array}$ & $\begin{array}{c}\text { Group II } \\
(n=28)\end{array}$ & $\begin{array}{c}\text { Group III } \\
(n=21)\end{array}$ \\
\hline Age (years) & $39.04(25-60)$ & $34.76(24-52)$ & $29.07(21-43) b^{* * *}, c^{*}$ \\
\hline Gender (F/M) & $73 / 61$ & $14 / 14$ & $11 / 10$ \\
\hline $\mathrm{BMI}\left(\mathrm{kg} / \mathrm{m}^{2}\right)$ & $26.95 \pm 5.6$ & $28.21 \pm 7.08$ & $24.75 \pm 3.68 c^{*}$ \\
\hline Smoking $(-/+)$ & $101 / 54$ & $28 / 0$ & $16 / 5$ \\
\hline Regular sleep (-/+) & $52 / 82$ & $12 / 16$ & $11 / 10$ \\
\hline Regular exercise $(-/+)$ & $111 / 23$ & $20 / 1$ & $1 / 20$ \\
\hline Vitamin supp. $(-/+)$ & $65 / 69$ & $13 / 8$ & $13 / 8$ \\
\hline \multicolumn{4}{|l|}{ Comorbidity } \\
\hline CVD $(-/+)(\%)$ & $91 / 43$ & $27 / 1$ & $2 / 19$ \\
\hline Diabetes mellitus $(-/+)$ & $100 / 34$ & $0 / 28$ & $3 / 18$ \\
\hline Asthma/allergy (-/+) & $94 / 40$ & $21 / 7$ & $2 / 19$ \\
\hline Viral load (high/low) & $67 / 67$ & $28 / 8$ & $17 / 4$ \\
\hline \multicolumn{4}{|l|}{ COVID-19 IgG efficacy } \\
\hline COVID-19 lgG levels & $7.15(0.19-12.8)$ & $9.36(4.58-2.41) a^{* *}$ & $7.34(0.01-12.5) c^{*}$ \\
\hline COVID-19 IgG (-/+) (\%) & $6 / 128(95.5)$ & 3/25 (89.3) & $0 / 21(100)$ \\
\hline
\end{tabular}

${ }^{*} p<0.05 ;{ }^{* *} p<0.01 ;{ }^{* * *} p<0.001$. a: between Groups I and III; b: between Groups I and II; c: between Groups II and III. Group I: two doses vaccinated individuals without COVID-19 disease or contact. Group II: two doses vaccinated individuals have had COVID-19 disease before. Group III: unvaccinated people who have had COVID-19. F: Female; M: male; BMI: body mass index; Vitamin supp.: vitamin supplementation; CVD: cardiovascular diseases. 
and spike proteins of SARS-CoV-2. Results were calculated as the ratio between the optical density of the sample and the optical density of the negative control in the kit and expressed as arbitrary units (AU). The sample positivity cut-off value for anti-nucleocapsid and/or anti-spike antibodies was determined by the manufacturer as $A U>1.1$.

Statistical analysis was performed using SPSS IBM 18.0 (SPSS Inc., Chicago, IL, USA). The distribution of the data was determined using the Kolmogorov-Smirnov test. The independent samples $t$-test was used to compare normally distributed variables, and the Mann-Whitney $U$ test was used to compare non-parametric distributed variables.

\section{RESULTS}

In the investigation of COVID-19 IgG antibody levels, a statistically significant difference was found between Groups I and II and between Groups II and III $(\mathrm{p}<0.01$ and $\mathrm{p}<0.05$, respectively). Antibody formation was highest in Group II. On the 21 st day after vaccination, the antibody positivity rate was $95.5 \%$ for Group I and $100 \%$ for Group II. The antibody positivity rate was $89.3 \%$ in Group III, who were not vaccinated (Table 1).

Group I was subgrouped according to age, gender, BMI, smoking, vitamin use, and working in areas with high viral load and comorbid diseases, and the relationship with antibody levels was evaluated. Mean antibody levels were $8.91(n=29,[1.98-$ $12.80])$ in the 20-30 age group, $6.86(\mathrm{n}=85,[0.19-12.78])$ in the $31-50$ age group, and $5.46(\mathrm{n}=20,[0.68-10.51])$ in the $>50$ age group. Antibody response levels were significantly higher in the 20-30 age group compared with the $31-50$ and $>50$ age groups ( $\mathrm{p}<0.01$ for both) (Figure $1 \mathrm{~A})$. In BMI subgroups, antibody levels were significantly higher in normal-weight subjects $(\mathrm{n}=60,[8.36(1.09-12.80)])$ compared with the overweight $(\mathrm{n}=39,[6.78(1.31-12.78)])$ and obese groups $(\mathrm{n}=35,[5.47$ $0.19-11.7])(\mathrm{p}<0.01$ for both) (Figure 1B).

Antibody response levels of those in Group I working in areas with high viral load were significantly higher compared with those working in areas with low viral load $(\mathrm{n}=66,8.31$ [1.40-12.80]; $\mathrm{n}=67,6.01$ [0.19-12.11]; respectively, $(\mathrm{p}<0.001)$ (Figure 2).

Antibody response levels were significantly lower in participants with comorbid diseases in Group I ( $\mathrm{n}=95,6.2$ [0.1912.26]) compared with those without comorbid diseases $(\mathrm{n}=39$, $9.45[1.98-12.80])(\mathrm{p}<0.001)$. Antibody levels were significantly lower in individuals with CVD compared with those without CVD ( $\mathrm{n}=43,6.16$ [1.40-12.80]), ( $\mathrm{n}=91,7.61$ [0.19-12.26]), respectively; $\mathrm{p}<0.05$ ). People with $\mathrm{DM}$ had significantly lower antibody response levels compared with those without DM $(\mathrm{n}=34,4.89$ [0.19-10.74]; $\mathrm{n}=100,7.92$ [1.21-12.8], respectively; $\mathrm{p}<0.001$ ) (Figure 2).

A negative correlation was found between IgG antibody response and BMI and age in Group I $(r=-0.336, p<0.001$ and $\mathrm{r}=-0.307, \mathrm{p}<0.001$, respectively).

\section{DISCUSSION}

Group II had the highest IgG antibody response after vaccination. Antibody levels of the vaccinated PCR (+) group were higher than the PCR $(+)$ group without vaccination $(\mathrm{p}<0.05)$. Similar to our findings, Jabal et al. ${ }^{5}$ showed that in single-dose BNT162b2 mRNA COVID-19 vaccination, post-vaccination

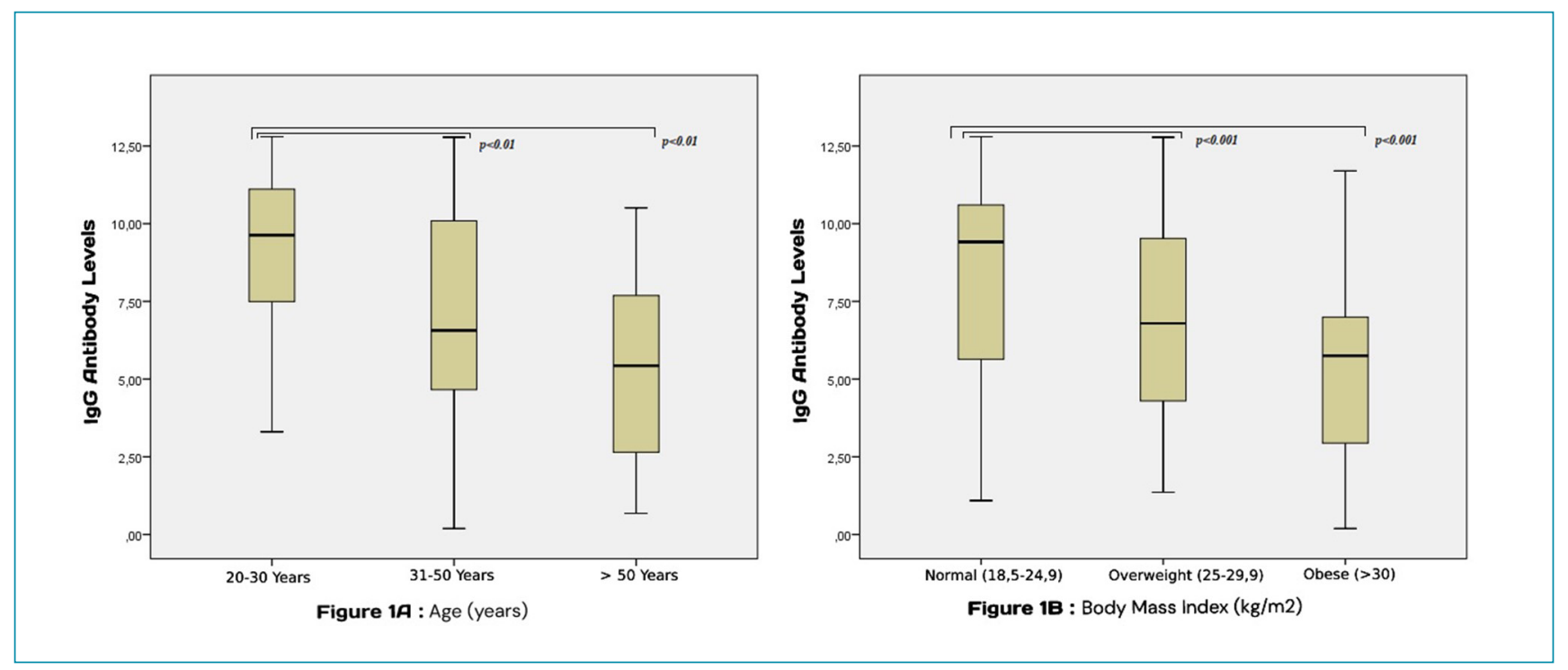

Figure 1. COVID-19 IgG levels in age (A) and body mass index (B) sub-groups of vaccinated Group 1, Eda Celik Guzel. 


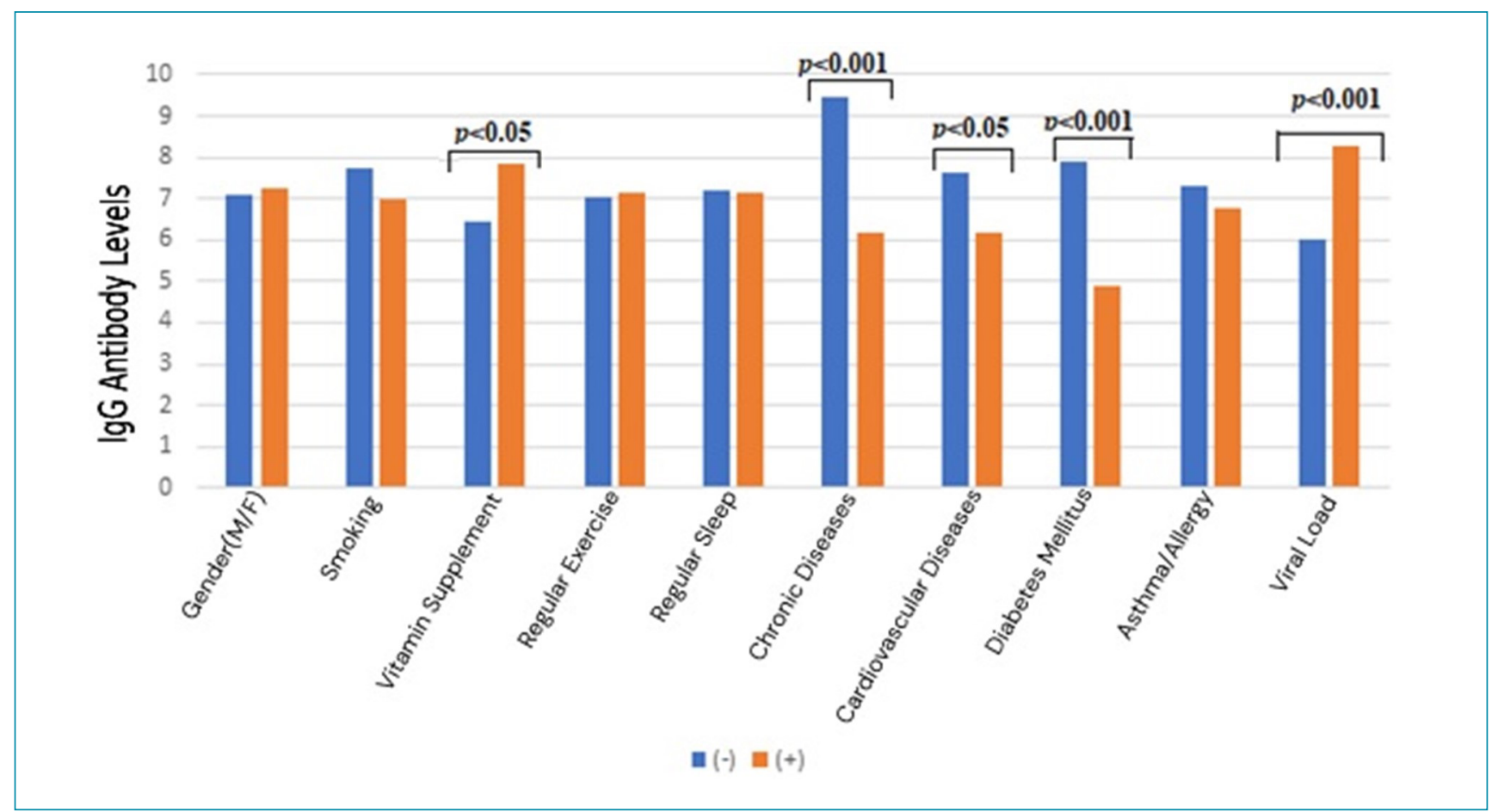

Figure 2. Differences of COVID-19 IgG antibodies Levels in sub-groups of vaccinated Group I, Eda Celik Guzel.

COVID-19 IgG antibody levels were much higher among those with previous evidence of disease. Furthermore, the antibody levels after vaccination in Group I reached values nearly equal to Group III (Table 1). It is a remarkable result that on the 21 st day after vaccination, the antibody positivity rate of Group I (95.5\%) was higher than that of Group III (89.3\%) (Table 1). These results confirm that the effectiveness of the CoronaVac vaccine is very high. At the same time, our findings were consistent with the results in the CoronaVac phase two study ${ }^{4}$.

Studies examining the effect of risk factors and comorbidities on antibody titer in vaccinated persons are quite limited. In this study, when Group I was subgrouped according to age, antibody levels were significantly higher in the 20-30 age group compared with that in the $31-50$ and over 50 age groups $(\mathrm{p}<0.01$ for both) (Figure 1A). Contrary to these results, preliminary results of the CoronaVac phase 1 study reported that antibody responses did not differ between the ages of 18 and 59 years ${ }^{2}$. CoronaVac phase two results also reported that antibody response did not differ in individuals over 60 years old, similar to the phase 1 study. Similar to our results, vaccine studies other than CoronaVac in the literature report that antibody formation decreases with age. Antibody titers were reported to decrease with age after a single dose of BNT162b2 mRNA COVID-19 vaccination of health care workers in Israel $(\mathrm{p}<0.001)$. Xia $S$ et $\mathrm{al}^{3}{ }^{3}$ found that the neutralizing antibody response against SARS-CoV-2 after BBIBP-CorV vaccination was lower in the
$>60$ age group compared with the 18-59 age group. In the examination of age, the antibody response was decreased in older age groups, which supports our study results ${ }^{6,7}$. In the evaluation of other vaccination studies, a negative correlation was detected between COVID-19 IgG antibody levels and age as this study supports this finding.

COVID-19 IgG antibody levels of normal-weight individuals in Group I were significantly higher than overweight and obese individuals $(\mathrm{p}<0.01$ for both) (Figure 1B). Therefore, it was found that antibody response decreases with increasing BMI. This result can be interpreted as either the insufficiency of the immune mechanisms in obesity or the insufficient antibody response of the vaccine dose in obese individuals. A similar finding has not yet been reported. In the investigation of obesity, Aude Richard et al. ${ }^{6}$ reported no significant relationship between BMI and seropositivity. However, they reported that obese women tended to have higher seropositivity compared with normal BMI. The existence of a negative correlation between IgG antibody levels and BMI in this study suggests that this mechanism may be different during the vaccination process.

There is a linear relationship between maintaining a healthy life and immune response ${ }^{8}$. Antibody levels were significantly higher in vitamin $\mathrm{C}$ and vitamin D users $(\mathrm{p}<0.05)$ (Figure 2). During the COVID-19 pandemic, the use of nutrients that strengthen immunity, such as vitamins $\mathrm{C}$ and $\mathrm{D}$, zinc, etc., increased. However, studies supporting the effect of dietary 
supplements in the prevention of COVID-19 are not clear and convincing'. In this study, $51.5 \%$ of the volunteers in Group I were taking vitamin supplements.

Another remarkable point is that among the health care professionals in Group I, the antibody levels of those working in areas with high viral load (i.e., emergency clinic, pandemic clinic, and pandemic ward) were significantly higher than those who did not work in these areas $(\mathrm{p}<0.001)$ (Figure 2). According to this result, it was thought that encountering a low viral load would keep the immune response ready, and a better antibody response can be generated after vaccination. In fact, Jabal et al. ${ }^{10}$ found that health care workers with previous evidence of infection had higher post-vaccination IgG levels, which supports this finding. Compared with the antibody response following the recovery of patients with COVID-19, a history of close contact with a person infected with SARS-CoV-2 was found to increase the probability of seropositivity approximately five times ${ }^{6}$.

There were no studies on the COVID-19 vaccine in the literature investigating the effects of comorbid diseases on antibody responses. Antibody levels were significantly lower in participants who had comorbid diseases in Group I $(\mathrm{p}<0.001)$. At the same time, antibody levels were significantly lower in individuals with CVD $(\mathrm{p}<0.05)$ and DM $(\mathrm{p}<0.001)$ (Figure 2$)$. However, antibody response following the recovery of patients with COVID-19 was investigated in various comorbid conditions, and it was reported that those with comorbidities had higher IgG titers two months after recovery ${ }^{11,12}$. In contrast, Meitian Yan et al. ${ }^{13}$ did not find any difference in the rate of positive IgG antibodies in the comorbidity group.

\section{Limitations}

This study has some limitations. ELISA tests can lead to false-positive results due to cross-reactivity with antibodies against other seasonal human coronaviruses ${ }^{14}$. To better interpret the results, it is necessary to validate the findings with larger case series. In addition, having the PCR results of people who were vaccinated in Group I could, of course, strengthen our results further.

\section{CONCLUSIONS}

IgG antibody seropositivity was found to be $95.5 \%$ in people who did not have COVID-19 and received CoronaVac vaccination. It was found that COVID-19 IgG antibody levels decreased with increasing age and BMI. Antibody levels were found to be higher in health care workers working in environments with high viral load compared with others. The presence of comorbidities (i.e., DM and CVD) decreased COVID-19 $\mathrm{IgG}$ antibody levels. As a result of this study, new information has emerged that will shed light on future studies with larger case series. It is evident that vaccination doses and repetitions should be reviewed especially in terms of age, obesity, and comorbidities.

\section{AUTHORS" CONTRIBUTION}

EÇG: Conceptualization, Formal analysis, Writing - original draft. AÇ: Data curation, Formal analysis, Writing - original draft. BE: Data curation, Formal analysis. NS: Data curation.

\section{REFERENCES}

1. World Health Organization. Coronavirus disease (COVID19) situation reports in Bnagladesh. Geneva: World Health Organization; 2020. [cited on Mar. 20, 2021]. Available from: https://www.who.int/emergencies/diseases/novelcoronavirus-2019/situation-reports

2. Zhang Y, Zeng G, Pan H, Li C, Hu Y, Chu K, et al. Safety, tolerability, and immunogenicity of an inactivated SARS-CoV-2 vaccine in healthy adults aged 18-59 years: a randomised, double-blind, placebocontrolled, phase 1/2 clinical trial. Lancet Infect Dis. 2021;21(2):18192. https://doi.org/10.1016/S1473-3099(20)30843-4

3. Xia S, Zhang $Y$, Wang $Y$, Wang $H$, Yang $Y$, Gao GF, et al. Safety and immunogenicity of an inactivated SARS-CoV-2 vaccine, BBIBP-CorV: a randomised, double-blind, placebo-controlled, phase 1/2 trial. Lancet Infect Dis. 2021;21(1):39-51. https:// doi.org/10.1016/S1473-3099(20)30831-8

4. Wu Z, Hu Y, Xu M, Chen C, Yang W, Jianget Z, al. Safety, tolerability, and immunogenicity of an inactivated SARS-CoV-2 vaccine (CoronaVac) in healthy adults aged 60 years and older: a randomised, double-blind, placebo-controlled, phase $1 / 2$ clinical trial. Lancet Infect Dis. 2021;21(6):803-12. https://doi. org/10.1016/S1473-3099(20)30987-7
5. Abu Jabal K, Ben-Amram H, Beiruti K, Batheesh $Y$, Sussan C, Zarka S, et al. Impact of age, ethnicity, sex and prior infection status on immunogenicity following a single dose of the BNT162b2 mRNA COVID-19 vaccine: real-world evidence from healthcare workers, Israel, December 2020 to January 2021. Euro Surveill. 2021;26(6):2100096. https://doi. org/10.2807/1560-7917.ES.2021.26.6.2100096

6. Richard A, Wisniak A, Perez-Saez J, Garrison-Desany $H$, Petrovic D, Piumatti G, et al. Seroprevalence of antiSARS-CoV-2 IgG antibodies, risk factors for infection and associated symptoms in Geneva, Switzerland: a populationbased study. medRxiv 2020:20248180. https://doi. org/10.1101/2020.12.16.20248180

7. Stringhini S, Wisniak A, Piumatti G, Azman AS, Lauer SA, Baysson $\mathrm{H}$, et al. Seroprevalence of anti-SARS-CoV-2 IgG antibodies in Geneva, Switzerland (SEROCOV-POP): a population-based study. Lancet. 2020;396(10247):313-9. https://doi.org/10.1016/ S0140-6736(20)31304-0

8. Alkhatib A. Antiviral Functional Foods and Exercise Lifestyle Prevention of Coronavirus. Nutrients. 2020;12(9):2633. https:// doi.org/10.3390/nu12092633 
9. Hamulka J, Jeruszka-Bielak M, Górnicka M, Drywień ME, ZielinskaPukos MA. Dietary supplements during covid-19 outbreak. Results of google trends analysis supported by plifecovid-19 online studies. Nutrients. 2020;13(1):54. https://doi.org/10.3390/nu13010054

10. Karimzaei T, Masoudi Q, Shahrakipour M, Navidiyan A, Jamalzae AA, Zoraqi Bamri A. Knowledge, attitude and practice of carrier thalassemia marriage volunteer in prevention of major thalassemia. Glob J Health Sci. 2015;7(5):364-70. https://doi. org/10.5539/gjhs.v7n5p364

11. Barin B, Yoldascan BE, Savaskan F, Ozbalikci G, Karaderi T, Çakal H. Joint investigation of 2-month post-diagnosis igg antibody levels and psychological measures for assessing longer term multi-faceted recovery among covid-19 cases in northern cyprus. Front Public Health. 2021;8:590096. https://doi.org/10.3389/fpubh.2020.590096
12. Kutsuna $S$, Asai $Y$, Matsunaga A, Kinoshita N, Terada $M$, Miyazato $Y$, et al. Factors associated with anti-SARS-CoV-2 IgG antibody production in patients convalescing from COVID-19. J Infect Chemother. 2021;27(6):808-13. https:// doi.org/10.1016/j.jiac.2021.01.006

13. Yan M, Zheng Y, Sun Y, Wang L, Luan L, Liu J, et al. Analysis of the diagnostic value of serum specific antibody testing for coronavirus disease 2019. J Med Virol. 2021;93(1):441-7. https://doi.org/10.1002/jmv.26230

14. Takahashi S, Greenhouse B, Rodríguez-Barraquer I. Are seroprevalence estimates for severe acute respiratory syndrome Coronavirus 2 biased? J Infect Dis. 2020;222(11):1772-5. https://doi.org/10.1093/infdis/jiaa523 